\title{
BMJ
}

\section{Preserving professional credibility: grounded theory study of medical trainees' requests for clinical support}

\author{
Tara J T Kennedy, research fellow, developmental paediatrician, ${ }^{1,2,3}$ Glenn Regehr, Richard and Elizabeth \\ Currie chair in health professions education research and professor, scientist, associate director, ${ }^{4,5,1} \mathrm{G}$ Ross \\ Baker, professor of health policy, management and evaluation, ${ }^{4}$ Lorelei Lingard, scientist, associate \\ professor, BMO financial group professor and education scientist ${ }^{1,6,7,8}$
}

\begin{abstract}
'Wilson Centre for Research in Education, Toronto, ON, Canada

${ }^{2}$ Bloorview Kids Rehab, Toronto, ON, Canada

${ }^{3}$ Stan Cassidy Centre for Rehabilitation, Fredericton, NB, Canada

${ }^{4}$ Faculty of Medicine, University of Toronto, Toronto, ON,Canada

${ }^{5}$ Toronto General Research Institute, University Health Network, Toronto, ON, Canada

${ }^{6}$ SickKids Learning Institute, Toronto, ON, Canada

${ }^{7}$ Department of Paediatrics, University of Toronto, Toronto, ON, Canada

${ }^{8}$ University Health Network, Toronto, ON, Canada

Correspondence to: T Kennedy tara.kennedy@utoronto.ca
\end{abstract}

Cite this as: BMJ 2009:338:b128 doi:10.1136/bmi.b128

\section{ABSTRACT}

Objective To develop a conceptual framework of the influences on medical trainees' decisions regarding requests for clinical support from a supervisor. Design Phase 1: members of teaching teams in internal and emergency medicine were observed during regular clinical activities (216 hours) and subsequently completed brief interviews. Phase 2: 36 in depth interviews were conducted using videotaped vignettes

to probe tacit influences on decisions to request support. Data collection and analysis used grounded theory methods.

Setting Three teaching hospitals in an urban setting in Canada.

Participants 124 members of teaching teams on general internal medicine wards and in the emergency department, comprising 31 attending physicians, 57 junior and senior residents, 28 medical students, and eight nurses. Purposeful sampling to saturation was conducted. Results Trainees' decisions about whether or not to seek clinical support were influenced by three issues: the clinical question (clinical importance, scope of practice), supervisor factors (availability, approachability), and trainee factors (skill, desire for independence, evaluation). Trainees perceived that requesting frequent/inappropriate support threatened their credibility and used rhetorical strategies to preserve credibility. These strategies included building a case for the importance of requests, saving requests for opportune moments, making a plan before requesting support, and targeting requests to specific team members.

Conclusions Trainees consider not only clinical implications but also professional credibility when requesting support from clinical supervisors. Exposing the complexity of this process provides the opportunity to make changes to training programmes to promote timely supervision and provides a framework for further exploration of the impact of clinical training on quality of care of patients.

\section{INTRODUCTION}

A central tenet of medical education is that trainees must progressively assume more responsibility for care of patients to become independent practitioners. ${ }^{1-6}$ In the course of assuming responsibility for such care, trainees regularly encounter situations that "exceed their confidence or skill to handle alone." In such situations, they have an "obligation to secure direct assistance from faculty or appropriately experienced residents." quality of care in clinical training depends, to a large part, on communication between trainees and supervisors in the form of requests for clinical help when required.

This reliance on trainees' requests works much of the time, but when it doesn't the results can be devastating. One infamous example of a breakdown in the supervision request process is the 2001 case at Boston Children's Hospital, where a state review determined that a 5 year old boy who died after a prolonged seizure did not receive optimal medical treatment. ${ }^{7}$ Two postgraduate medical trainees arrived soon after the seizure began, but a senior physician was not called to the bedside until an hour into the episode.

This case shows the link between quality of care and timely requests for clinical help. It is potentially dangerous to assume that trainees' requests for support arise directly from clinical need, without complication. Ethnographic studies of medical education and research on case presentations have suggested that communication between trainees and their supervisors is not a straightforward transmission of clinical information but rather is complicated by issues such as evaluation, learning agendas, and professional socialisation. ${ }^{8-11}$

If we are to continue to provide the educational benefit of clinical independence to trainees, ${ }^{12}$ a critical examination of the process of requesting clinical support is required. We developed a conceptual framework of the influences on trainees' decisions to request clinical help from a supervisor through exploration of teaching team practices.

\section{METHODS}

We designed the study using grounded theory, ${ }^{13-16} \mathrm{a}$ research method intended to develop a theoretical explanation of a social phenomenon that is "grounded in" (that is, derived from) naturalistic data. ${ }^{17}$ Participants 
in the study were 124 members of clinical teaching teams in general internal medicine and emergency medicine, including attending physicians $(n=31)$, junior and senior residents $(\mathrm{n}=57)$, medical students $(\mathrm{n}=28)$, and nurses $(n=8)$. We used purposeful sampling ${ }^{13}$ to ensure inclusion of participants of both sexes and of different levels of experience. Sampling continued until saturation of the data was reached (the point at which further sampling ceases to yield any new analytical concepts). ${ }^{18}$

In the Canadian medical education system, students complete an undergraduate university degree before entering medical school. Medical school programmes last three to four years, the final two years of which are spent training primarily in clinical settings. The medical students in this study were all in their third or fourth year of medical school. Graduates of Canadian medical schools then enter postgraduate training programmes, commonly called residencies, before undertaking the certification examinations required to practise as independent physicians. These last from two to five or more years, depending on the degree of subspecialisation undertaken. The junior resident participants were in their first or second year of postgraduate training. The senior residents were all late in their second year of specialty training after medical school or beyond.

The project took place in three academic health sciences centres associated with a Canadian medical school in a large urban setting. Sampling from the three centres was performed to provide a broad range of relevant perspectives and practices and to increase transferability of resultant analytic concepts. Settings included the emergency departments and the general internal medicine inpatient teaching wards of the three centres. We chose these areas of intense clinical teaching to represent different clinical supervisory structures. In the emergency medicine settings, all trainees, regardless of seniority, assessed patients on their own and then reported back to an in house attending physician. On the general internal medicine wards, trainees worked in teams with at least one medical student, one or two junior residents, a senior resident, and an attending physician. The attending physicians and senior trainees provided supervision for junior trainees in a hierarchical supervisory structure, with the senior resident running the day to day clinical activities. On the general internal medicine wards, the senior resident was generally the senior in house physician at night, while the attending physician could be contacted at home for support. In both settings, the amount of direct contact between attending physicians and trainees was determined by the individual attending physician (and depended on several variables in the specific clinical context). ${ }^{19}$ Throughout the course of clinical training in Canada, attending physicians provide evaluation and feedback for all trainees under their supervision (with input from senior trainees when they are working in teams like in general internal medicine).

\section{Phases of study}

The study was designed in two phases. Phase 1 consisted of non-participant observations and brief interviews. Phase 2 consisted of in depth interviews with video vignette prompts. The principal investigator and a research assistant collected all data; neither had any affiliation with the study sites or previous knowledge of the participants.

Phase 1 involved non-participant observation ${ }^{20}$ of 12 teaching teams (seven in general internal medicine and five in emergency medicine, with a total of 88 team members observed). Each team was observed on six occasions over the course of one month (total of 216 hours of observation). Observations were scheduled to sample the maximum possible variety of clinical activities for each team. Detailed structured field notes were kept. ${ }^{20}$ Observers initially recorded, in as much detail as possible, any observed interactions between supervising physicians and trainees (including the content of conversations, the context, participants and intended audience for relevant comments, and the nonverbal nuances involved in the interactions). In accordance with grounded theory principles, the structure of the field notes evolved as the study progressed to reflect emergent analytical concepts. ${ }^{13}$ As a source of triangulating data, 65 members of teaching teams also completed a brief (15 minute) interview near the end of the month of observations (we did not interview trainees whose rotation with the team finished before the end of the observation period). The interviews were used to explore the authenticity ${ }^{21}$ of the observational data and to probe the intentions and rationales behind observed behaviours. The interviews were audiotaped and were anonymised during transcription.

Table 1|Clinical factors affecting requests from medical trainees for clinical support that point I was like 'this guy could get really sick really fast,' so I just ran out and called the fifth year emerg resident" (junior resident 13, emergency medicine) 
We analysed the field notes, reflective notes, and interview transcripts for emergent themes using grounded theory methods. ${ }^{17}$ Data collection and analysis proceeded simultaneously in an iterative fashion, in which the results of the ongoing data analysis informed subsequent data collection. Two researchers recursively read the dataset to develop a preliminary coding structure. ${ }^{13}$ The research team, which included a paediatric clinician educator (who had recently finished training), a qualitative education scientist, a cognitive psychologist, and a health policy researcher, discussed, refined, and confirmed this coding structure. Confirmability was ensured by maintaining an audit trail of all analytical memos, minutes of the meetings, and revisions to the coding structure. One coder applied the final coding structure to the complete dataset, using NVivo software to facilitate cross referencing. ${ }^{22}$ Phase 1 generated a model of supervision practices and an exploration of how supervisors make decisions about the nature of the supervision to be provided to clinical trainees, which has been published elsewhere. ${ }^{19}$ Phase 1 also produced a preliminary conceptual framework regarding decisions to request supervision that was expanded in phase 2 .

Phase 2 was designed to refine and expand the emerging conceptual framework, through in depth interviews with video prompts (a technique effective at probing unspoken behavioural influences). ${ }^{2324} \mathrm{We}$ developed a series of 10 videotaped vignettes (five set in general internal medicine and five in emergency medicine), each crafted to present a dilemma relevant to decisions about supervision. The vignettes were based on events that occurred during the observations (with details altered to render the original participants unidentifiable). For example, one vignette portrayed a junior resident deliberating about whether or not she should call her attending physician before giving a patient heparin in the middle of the night.

Phase 2 participants included 19 attending physicians, 13 residents, and four medical students. Although the interviews with attending physicians provided some contextual and confirmatory data that were relevant, we report here mainly on phase 1 data and the trainee interviews from phase 2. During the interviews, we asked participants to discuss their opinion of the appropriate response to the dilemma presented in each video vignette and to articulate other possible responses and their reasons for rejecting these (the discourse based interview method). ${ }^{25} \mathrm{We}$ analysed anonymised interview transcripts for both emergent themes and pre-selected themes that had emerged from the phase 1 data. We used an iterative and constant comparative method, in the grounded theory tradition, ${ }^{13}$ in analysis as described above.

\section{RESULTS}

Trainees' decisions about whether or not to seek clinical support were influenced, as one would expect, by the nature of the clinical situation in question, but also by factors related to supervisors and to the trainees themselves (tables 1-3). The more urgent the clinical situation or the more important the implications of the clinical decision to be made, the more likely trainees were to seek support from their supervisors. As one junior resident said: "I'll use as an example chest pain... if chest pain is getting to a point where the patient might need a higher level of care then I'll always ask for help earlier rather than later" (junior resident 14, general internal medicine). Trainees also considered whether or not the clinical question fell within the scope of practice that was expected of them at their level of training. They expressed reluctance to ask for help if the clinical situation was one that they perceived they should have mastered at their level. A senior resident explained: "If I want [attending physician] to watch me suture, then he probably would laugh at me because that is expected of me to know that" (senior resident 1, emergency medicine). On the other hand, trainees seemed to have an impression of the types of clinical situation that always warranted their contacting a supervisor. For example, one medical student relayed a situation where there was a question about a patient's discharge status: "I contacted [attending physician] because . . . obviously I cannot make the call of whether or not [patient's name] can stay in the hospital" (medical student 8, general internal medicine).

Decisions about whether or not to seek clinical support were affected not only by the clinical implications of their question. Trainees also considered issues related to their supervisors (table 2). At a practical level, they considered their supervisor's proximity and availability. When supervisors were perceived to be busy, or were not physically present and therefore potentially busy with something else, trainees thought

Table 2 | Supervisor factors affecting requests from medical trainees for clinical support

Themes and definitions

Availability

Refers to examples of how physical proximity of supervisor and timing of clinical situation affect likelihood that trainees will ask for clinical support

Refers to influence of trainees' perceptions of their relationship with supervisor on decisions about whether or not to ask for clinical support

\section{Representative transcript excerpts}

"Especially if they're busy, if they're running around, answering pages, reassessing people, trying to deal with EMS calling on the patch phone for example... it can make it tough to sort of stick your head in and say 'can we review a case' or 'I have a question'” (junior resident 9, emergency medicine)

"There are certain staff that are known for ... they don't like to be bothered, you feel that if you call them they'll feel it's just a petty problem, why didn't you look after it yourself. They'll think less of you as a resident" (junior resident 14 , general internal medicine) 
Table 3 | Trainee factors affecting requests from medical trainees for clinical support

\section{Expertise}

Refers to situations where trainees' level of expertise has an impact on their "I guess one of my pet-peeves ... there were sort of a bunch of things las ability to know when it would be appropriate to ask for clinical support year where it wasn't so much a problem of people didn't know how to manage patients, but they just didn't know how or when to call for help. An that's sort of when bad things can happen" (senior resident 8 , general internal medicine)

Desire for independence

Refers to instances where trainees' desire to take on responsibility and perform clinical activities independently might influence decisions about requesting clinical support

Evaluation and assessment

Refers to instances where trainees make decisions about requesting clinical support for evaluative or educational purposes

that their clinical question had to be sufficiently important to warrant a call for help. One senior resident said: "it's not so hard to ask anything if the staff is standing right there, but if it is 1.30 in the morning and your resident is asleep ... I think that has a big impact" (senior resident 3, emergency medicine). The trainees also considered their relationship with the supervisor when deciding whether or not to ask for supervision. Trainees often used the term approachability to refer to their perception of the ease with which they could ask a particular supervisor for support. As a senior resident explained: "You get a vibe from your staff very quickly on when or when you shouldn't ask for help. And some staff are very open and up-front: 'call me for anything' very approachable. And some staff you get the impression that if you call them in the middle of the night it's going to be a huge deal and they'll be talking in the morning and be sort of like 'I can't believe him. He called in the middle of the night' ..." (senior resident 10 , general internal medicine).

Finally, trainees' decisions about whether or not to seek clinical support were influenced by factors related to the trainees themselves (table 3). For example, trainees described how their desire for independence in clinical skills affected their decisions about seeking help from supervisors. As a junior resident said: "It is empowering when you know you can do that, empowering when you have a question and then you think about it and you make a decision and then you find out later if it is right" (junior resident 5 , general internal medicine).

Trainees also discussed how their concern about evaluation and assessment could affect decisions, as in the following excerpt from an interview with a senior resident in general internal medicine: "Evaluations. They figure into it. To get into med school everyone has to love you and you have to have straight As. To get into a residency programme of your choice, everyone has to love you and you have to have straight As. And so when you go through residency you're figuring you're still in that mode: 'Everyone has to love me and I have to get super high marks.' And evaluations are all subjective right? So you piss off the guy by waking him up and he's
"I think there is some personal ... satisfaction of getting a procedure done by yourself especially when you are a junior, you know it is really satisfying, so you might try not to ask for help" (senior resident 3, emergency medicine)

"I want to look like I'm independent and I can handle questions on my own and I don't need to go to the attending for every little thing unless it's big.. [because] you want to impress and you want to have good things said about you at the end of your rotations" (junior resident 5 , emergency medicine)

going to give you a bad evaluation. If that matters to you, you won't wake him up" (senior resident 6 , emergency medicine).

Trainees also realise that they might, in some cases, lack the expertise required to recognise when they cannot cope and need support from their supervisors. As one medical student put it: "Generally I won't ask for advice unless it seems urgent, which I'm not sure if I'm the best judge of that" (medical student 1, general internal medicine).

Many factors other than the clinical implications are therefore involved in decisions about requests for clinical support. Furthermore, we saw that trainees struggle with (and sometimes, particularly in the middle of the night, agonise over) the decision of whether or not to call their supervisor to ask for help. What makes these decisions so difficult and so complicated? The issue of credibility emerged from our data as central to this question. Trainees perceive that their professional credibility could be at risk when they admit to a supervisor that they need help. Trainees perceive that asking for "appropriate" support aids credibility (akin to "knowing one's limits"), but that asking too often or for too much support threatens their credibility as a practitioner. The fact that trainees use rhetorical strategies (persuasive arguments) ${ }^{26}$ to preserve their credibility when making requests for help supports this theory. We have used the term "rhetorical strategy" here in the theoretical sense to indicate language strategies that are purposeful, are targeted towards a particular audience, and serve a persuasive function. ${ }^{27} \mathrm{We}$ do not intend to invoke the derogatory lay meaning of rhetoric involving artificiality or deception. Trainees used four main rhetorical strategies to preserve their credibility when requesting clinical support from a supervisor.

\section{Building a case}

Along with their request for help, trainees often emphasise details that support the urgency or the importance of their clinical situation as a type of justification for making the request. For example, in the following excerpt from our observation field notes (box 1), a junior resident working in the emergency 
department asked her attending physician to see a patient before she finished taking the history, emphasising the patient's level of pain.

\section{Box 1 Transcript from field notes}

JR [junior resident] comes in to the nursing station-AP [attending physician] is talking to a nurse.

JR "Sorry ... would you come see the guy in 12 with me? He's in really a lot of pain, writhing around on the bed, I didn't think I should wait."

JR presents the case in a couple of sentences as they walk down the hallway.

Field notes, emergency medicine team 5 , day 1

\section{Saving questions}

Trainees saved questions and asked them at times convenient to their supervisor. Disturbing a supervisor for an "insignificant" question was perceived as a threat to credibility, while asking the same question of a supervisor who was close by and available did not have the same implications. Consider the excerpt shown in box 2 from our observation notes, in which a trainee's decision to "save" her request for help in interpreting imaging results leads to a difficult situation.

\section{Box 2 Transcript from field notes}

JR3 [junior resident 3]: "Can we review some imaging? All mine are back now."

AP [attending physician] and JR3 look at a CXR [chest $x$ ray picture]. They then review the old $x$ ray together.

Nurse comes up and asks for nausea meds for the patient with post-concussion syndrome.

JR3: "That's my patient-I was going to order Gravol [dimenhydrinate] and I forgot ... I'll do that [writes orders on the chart]. I got the $x$ ray of the spine and then the CT head."

AP: "We were going to see the c-spine [cervical spine] $x$ ray before the CT to decide if we needed a CT [computed tomogram] of the spine."

Junior resident 3: "Oops ..." [with grimace].

AP looks at the c-spine xrays for a long time. (There is a sense that they are on the borderline of being adequate to rule out a spine injury and that $\mathrm{AP}$ might have gotten the $\mathrm{CT}$ spine if the patient hadn't already gone up for CT head).

Attending physician: "I think they're OK."

Junior resident 3: "Sorry I jumped the gun on the CT."

Field notes, emergency medicine team 5 , day 4

\section{Making a plan}

Trainees preserved credibility by making plans and then checking with a supervisor, rather than asking an open ended question about what to do. A senior resident explained: "Sometimes when I've had questions, and really not been familiar with the area that my question is on, I will often try to gather some information before I approach my supervisor so I don't seem like I don't know anything"' (senior resident 3, emergency medicine).
Attending physicians confirmed the impact of this strategy on a trainee's assessment, as in the following representative excerpt: "A good trainee will say 'Here's the information. Here's my impression and my plan. Can you check this before I implement it?' And that's the optimal trainee. That's someone who's said 'Here I go, I've made an effort to do everything I can, but because I'm not $100 \%$ comfortable I would like you to check and see if you agree.' But not everybody does. Some people either go ahead and do it and then ask you, and you find out after or you see in the ward or written on the chart that they've done something potentially dangerous. Or you have someone who doesn't even make an effort to come up with any plan, but just wants you to make a plan" (attending physician 15, general internal medicine).

\section{Targeted questions}

Trainees often targeted questions to less "powerful" members of the team, such as junior residents or allied health professionals, to avoid exposing a lack of clinical knowledge or skill to a supervisor. In this example (box 3), a junior resident asked for clinical information from a peer before speaking with her senior resident.

\section{Box 3 Transcript from field notes}

As they are waiting for SR [senior resident], JR1 and JR2 discuss a lab result from a patient on the floor (INR [international normalised ratio] is very high). JR1 mentions that she will obviously hold the Coumadin [warfarin] (the medication that caused the ratio to be raised) and asks JR2 what else he would do. JR2 suggests giving vitamin K. They agree that they would not give FFP [fresh frozen plasma] unless the patient was bleeding actively... JR2 then leaves to go see his patient, and JR1 waits for SR.

JR1: "The INR is 10.99."

SR: "Wow!"

JR1 gives a couple of other pertinent lab results.

JR1: "I'll hold the Coumadin."

SR: "And my next question is ..."

JR1: "Is she bleeding—no. I would give her vitamin K."

SR: "Yes. I wouldn't give FFP unless she bleeds."

JR1 goes to take care of the situation.

Field notes, general internal medicine team 7 , day 5

\section{DISCUSSION}

During clinical training, when trainees experience progressive independence in the care of patients, one of the main safety mechanisms is the trainees' obligation to ask for clinical help from a supervisor when faced with problems that exceed their clinical knowledge and skills. We have shown the complexity of the process of requesting support and the strategies trainees use to maintain professional credibility when making such requests. Trainees consider the preservation of their professional credibility along with the clinical implications of their situation when deciding about whether and 
how to ask for support. Training programmes and clinical educators should not take for granted timely requests for clinical support.

The fact that trainees are concerned about their professional credibility during communication with their supervisors does not indicate that they are unconcerned about patients' wellbeing. In fact, many trainees said that concerns about patients' care would trump concerns about credibility. As one junior resident in general internal medicine said: "you realise that if you make the wrong decision you can cause serious harm to someone. So you get over looking stupid really quickly and just ask for help" (junior resident 4, general internal medicine). Trainees' concern about professional credibility also involves important learning issues. The desire to develop independence in clinical reasoning and the desire to promote an evolving identity as an independent clinician are both appropriate educational pursuits. Thus trainees' consideration of professional credibility when making decisions about asking for help is not necessarily inappropriate, but it does raise issues of practical importance to the safety of patients on clinical teaching teams.

\section{Implications for medical education practice}

Many of our participants believed that patients' safety in clinical teaching situations could be improved by explicitly addressing the link between credibility and asking for help. One attending physician stated: "I have to break them ... it's hard, but by the end of the rotation they are getting it. I say over and over, it's ok to say I don't know. . ." (attending physician 12, emergency medicine).

We have provided a framework for several interventions that might improve transparency in supervisor-trainee communication about supervision. Trainees could be explicitly taught rhetorical strategies such as "planning before asking" and "targeting questions" to increase their comfort with requesting support from a supervisor. An explicit "scope of practice" could be defined for trainees in a given context to promote a shared understanding of the types of situation that would always warrant contacting a supervisor (such as end of life discussions for junior trainees) or warrant seeking expert help (such as clinical criteria for accessing a rapid response team). ${ }^{2829}$ Requests for assistance could be explicitly included in the clinical evaluation process, with feedback provided on performance of this skill. The impact of such interventions on the teaching team environment and quality of patients' care will be important to evaluate.

Our results are also important for clinical supervisors who want to maximise the quality of the clinical care provided by their teaching teams. An understanding of the factors involved in trainees' decisions about requesting support allows the management of these factors to promote clinical supervision. To give a simple example, consider the issue of availability of a supervisor. Our dataset was filled with instances where trainees reported that they considered asking for help but decided against it because their supervisor wasn't close by, and they thought that their question wasn't important enough to warrant a phone call to someone who might be busy with something else. Addressing the issue of supervisor proximity by increasing the number of times supervisors and trainees meet during the day might promote timely discussion about trainees' clinical concerns. An evaluation of the effects of such changes to supervision practices on care outcomes would lay the groundwork for the necessary, but as yet largely neglected, study of the links between supervision practices and quality of care. ${ }^{12}$

Our study shows that asking for help from a supervisor involves a complex decision making process for medical trainees. This leads to a question of key importance to medical educators: how and why has a medical training culture evolved in which asking for help can be so difficult? Medical anthropologists such as Bosk $^{30}$ and Sinclair ${ }^{11}$ have described how factors like professional responses to error ${ }^{30}$ and evaluation processes ${ }^{11}$ can contribute to a lack of transparency in communication between medical trainees and their supervisors. Further exploration of this phenomenon from a sociocultural perspective will be required better to understand, and ultimately influence, the multifactorial contributors to the generation of a medical training culture in which asking for help is sometimes problematic for trainees.

\section{Limitations}

Our results should be interpreted with observer effect ${ }^{31}$ and transferability in mind. ${ }^{32}$ We took two important measures to minimise the impact of observer effect on the results. Firstly, a consistent researcher (the principal investigator or a research assistant, both unknown to participants before the study) conducted observations for periods of three hours or more, so that team members could get used to the observer's presence. Secondly, participants were not made aware of the specific focus on supervision until after the observations were completed (participants consented to having all discussions between attending physicians and trainees recorded) to prevent them from altering this specific aspect of their work. With respect to transferability, it is important to consider that these data were collected in an urban setting and on medical services. The fact that the reported themes were common across the two distinct clinical settings (general internal medicine and emergency medicine), the three separate institutions with different supervision policies and practices, and the broad range of participants within each setting supports the transferability of the analysis. It remains to be tested, however, whether the theory of credibility will be relevant in different settings (such as surgical services or rural teaching practices).

\section{Conclusions}

Medical trainees consider not only clinical implications but many other factors when requesting support from a supervisor. Requests for support are perceived by trainees as potential threats to their professional credibility, and trainees thus use several rhetorical 


\section{WHAT IS ALREADY KNOWN ON THIS TOPIC}

The quality of clinical care provided to patients by medical trainees depends on the assumption that trainees can and will request clinical support from their supervisors when required

Communication between medical trainees and their supervisors is complicated by issues such as evaluations and learning agendas

\section{WHAT THIS STUDY ADDS}

Interview and observational data from clinical teaching teams showed that trainees' decisions about requesting support from clinical supervisors are complex

Trainees consider not only clinical implications but also personal credibility when making requests for clinical support

Exposure of factors affecting medical trainees' requests for clinical support provides the opportunity to make changes to clinical training programmes to promote timely clinical oversight by supervisors
7 Pope J. Even top hospitals make mistakes. CBS News 2003, September 29. www.cbsnews.com/stories/2003/09/29/ health/main575546.shtml

8 Lingard L, Garwood K, Schryer CF, Spafford MM. A certain art of uncertainty: case presentation and the development of professional identity. Soc Sci Med 2003;56:603-16.

9 Sommers PS, Muller JH, Saba GW, Draisin JA, Shore WB. Reflections-onaction: medical students' accounts of their implicit beliefs and strategies in the context of one-to-one clinical teaching. Acad Med 1994;69:S84-6

10 Konner M. Becoming a doctor. New York: Viking Penguin, 1987.

11 Sinclair S. Making doctors. Oxford: Oxford International Publishers, 1997.

12 Kennedy TJ, Regehr G, Baker GR, Lingard LA. Progressive independence in clinical training: a tradition worth defending? Acad Med 2005;80:S106-11.

13 Glaser B, Strauss A. The discovery of grounded theory: strategies for qualitative research. Chicago: Aldine, 1967.

14 Kennedy TJ, Lingard LA. Making sense of grounded theory in medical education. Med Educ 2006;40:101-8.

15 Charmaz K. Grounded theory: objectivist and constructivist methods. In: Denzin N, Lincoln Y, eds. Handbook of qualitative research. Thousand Oaks, CA: Sage, 2000:509-35.

16 Charmaz K. Grounded theory in the 21st century: applications for advancing social justice studies. In: Denzin N, Lincoln Y, eds. The Sage handbook of qualitative research. Thousand Oaks, CA: Sage, 2005:507-36.

strategies to preserve credibility during these requests The exposure of the complexity of this taken for granted process provides the opportunity to make changes to training programmes that might promote timely discussion of trainees' clinical concerns and also provides a framework for necessary further exploration of the impact of clinical training practices on quality of patient care.

We thank Douglas Buller of the Wilson Centre, University of Toronto, for his technical expertise in the production of the videos, and Lisa Kitchen of the University of Ontario Institute of Technology for her invaluable work in data collection and study coordination.

Contributors: All authors contributed to the conception and design of the study, the analysis and interpretation of the data, critical revision of the manuscript, and obtaining funding for the study. They are all guarantors. TJTK acquired the data and drafted the manuscript.

Funding: This study was supported by an operating grant from the Canadian Institutes of Health Research (CIHR), which had no involvement in the research process. LL is supported by a CIHR New Investigators Award. Competing interests: None declared.

Ethical approval: This study was approved by the institutional review board, and informed consent was given by all participants.

1 American Association of Medical Colleges. Compact between resident physicians and their teachers.

2006. www.aamc.org/meded/residentcompact/

2 AAMC policy guidance on graduate medical education: assuring quality patient care and quality education. Acad Med 2001;78:112-6.

3 Royal College of Physicians and Surgeons of Canada. General standards of accreditation.

2002. http://rcpsc.medical.org/publications/index.php

4 General Medical Council. The new doctor. London: GMC, 1997.

5 General Medical Council. Standards of training for the foundation programme.

2007. www.gmc-uk.org/education/documents/

Standards_for_Training_270307.pdf

6 Australian Medical Association. AMA position statement: prevocational medical education and training.

2005. www.ama.com.au/web.nsf/doc/WEEN-6JVTW2 